cers. Notably, mining the Oncomine database additionally revealed significant overexpression of both TYRO3 and MER in various B- and T-cell leukemia/lymphoma datasets, but not in AML (data not shown). This might suggest a potentially different mechanism of oncogenic signaling in lymphoid neoplasms involving all three receptor family members, as opposed to the clear role of AXL in FLT3-ITD ${ }^{+}$ $\mathrm{AML}$; this could be a focus point for further investigations.

The new study by Dumas et al. described a resistance mechanism against the FLT3 inhibitor quizartinib, where both the FLT3-ITD mutation and the GAS6-AXL axis trigger important kinase signaling cascades. Their results suggest that a combination of FLT3- and AXL-specific inhibitors, or the exploitation of dual FLT3-AXL inhibitors (e.g. cabozantinib), might be beneficial in FLT3-ITD ${ }^{+}$AML patients at risk of relapse. Furthermore, selective STAT5 inhibitors have the potential to become an effective tool for targeted and combinatorial therapy in AML.

In summary, FLT3 inhibitors display efficacy in the treatment of FLT3-ITD-driven AML, but more efficient targeting of AML blasts remains an unresolved medical need to fight therapeutic resistance, and to improve the poor overall survival and quality of life of patients.

\section{Funding}

HAN and RM are supported by the Austrian Science Fund (FWF) [SFB-F04707, SFB-F06105, and under the frame of ERA PerMed (I 4218-B) and ERA-NET (I 4157-B)]. RM, HAN and $A O$ were also generously supported by a private donation from Liechtenstein.

\section{References}

1. Daver N, Schlenk RF, Russell NH, et al. Targeting FLT3 mutations in AML: review of current knowledge and evidence. Leukemia. 2019;33(2):299-312.

2. Dumas PY, Naudin C, Maetin-Lannerée S, et al. Hematopoietic niche drives FLT3-ITD acute myeloid leukemia resistance to quizartinib via STAT5- and hypoxia- dependent up-regulation of AXL.
Haematologica. 2019;104(10):2017-2027.

3. Kirane A, Ludwig KF, Sorrelle N, et al. Warfarin Blocks Gas6-Mediated Axl Activation Required for Pancreatic Cancer Epithelial Plasticity and Metastasis. Cancer Res. 2015;75(18):3699-3705

4. Wu X, Liu X, Koul S, et al. AXL kinase as a novel target for cancer therapy. Oncotarget. 2014:5(20):9546-9563.

5. Lee HJ, Jeng YM, Chen YL, et al. Gas6/Axl pathway promotes tumor invasion through the transcriptional activation of Slug in hepatocellular carcinoma. Carcinogenesis. 2014;35(4):769-775.

6. Holstein E, Binder M, Mikulits W, et al. Dynamics of Axl Receptor Shedding in Hepatocellular Carcinoma and Its Implication for Theranostics. Int J Mol Sci. 2018;19(12).

7. Linger RMA, Keating AK, Earp HS, et al. TAM Receptor Tyrosine Kinases: Biologic Functions, Signaling, and Potential Therapeutic Targeting in Human Cancer. Adv Cancer Res. 2008;100:35-83

8. Paolino M, Penninger JM. The Role of TAM Family Receptors in Immune Cell Function: Implications for Cancer Therapy. Cancers (Basel). 2016;8(10).

9. Yoshioka T, Shien K, Takeda T, et al. Acquired resistance mechanisms to afatinib in HER2-amplified gastric cancer cells. Cancer Sci. 2019 Jun 4. [Epub ahead of print]

10. Mizuki M, Schwable J, Steur C, et al. Suppression of myeloid transcription factors and induction of STAT response genes by AML-specific Flt3 mutations. Blood. 2003;101(8)3164-3173.

11. Nelson EA, Walker SR, Xiang M, et al. The STAT5 Inhibitor Pimozide Displays Efficacy in Models of Acute Myelogenous Leukemia Driven by FLT3 Mutations. Genes Cancer. 2012;3(7-8):503-511.

12. Wingelhofer B, Maurer B, Heyes EC, et al. Pharmacologic inhibition of STAT5 in acute myeloid leukemia. Leukemia. 2018;32(5):1135-1146.

13. Yoshimoto G, Miyamoto T, Jabbarzadeh-Tabrizi S, et al. FLT3-ITD upregulates MCL-1 to promote survival of stem cells in acute myeloid leukemia via FLT3-ITD-specific STAT5 activation. Blood. 2009:114(24):5034-5043.

14. Maurer B, Nivarthi H, Wingelhofer B, et al. High activation of STAT5A drives peripheral T-cell lymphoma and leukemia. Haematologica. 2019 May 23. [Epub ahead of print]

15. Pham HTT, Maurer B, Prchal-Murphy M, et al. STAT5BN642H is a driver mutation for T cell neoplasia. J Clin Invest. 2018;128(1):387-401

16. Bowman RL, Busque L, Levine RL, et al. Clonal Hematopoiesis and Evolution to Hematopoietic Malignancies. Cell Stem Cell. 2018;22(2):157-170.

17. Fuster JJ and Walsh K. Somatic Mutations and Clonal Hematopoiesis. Circ Res. 2018;122(3):523-532.

18. Nogami A, Oshikawa G, Okada K, et al. FLT3-ITD confers resistance to the PI3K/Akt pathway inhibitors by protecting the mTOR/4EBP1/Mcl-1 pathway through STAT5 activation in acute myeloid leukemia. Oncotarget. 2016;6(11):9189-9205.

19. Rhodes DR, Yu J, Shanker K, et al. ONCOMINE: a cancer microarray database and integrated data-mining platform. Neoplasia. 2004;6(1):1-6.

\title{
Pediatric aplastic anemia treatment patterns and responses; power in the numbers
}

\author{
Lucy C. Fox ${ }^{1,2}$ and David S. Ritchie ${ }^{2,3}$
}

${ }^{1}$ Diagnostic Haematology, Peter MacCallum Cancer Centre; ${ }^{2}$ University of Melbourne and ${ }^{3}$ Clinical Haematology, Peter MacCallum Cancer Centre and Royal Melbourne Hospital, Melbourne, Australia

E-mail: DAVID S. RITCHIE - david.ritchie@mh.org.au

doi:10.3324/haematol.2019.225870

I n this edition of Haematologica, Rogers et al., representing 25 individual institutions, collectively report on their findings of the diagnostic approaches, applied therapies and responses in a cohort of 314 pediatric patients (aged 1-20 years) with a diagnosis of aplastic anemia (AA) collected through the North American Pediatric Aplastic Anemia Consortium (NAPAAC). ${ }^{1}$ This study highlights a number of important messages; specifically the power of collated registry data in a rare disease, the need to retest and continually refine diagnostic crite- ria to be fit for real-world purposes, the patterns of response and relapse to immunosuppressive therapy (IST) in a pediatric setting including the substantial differences in outcomes from IST in children compared with that in adults, and the importance of allogeneic stem cell transplant (HCT) in the therapy of refractory or relapsed disease.

Although AA can affect people at any stage of life, with a well reported bimodal peak of age incidence observed in older children/young adults and those over sixty years, 
it is a rare condition that often poses a diagnostic dilemma with acquired AA potentially confounded by a differential of inherited bone marrow failure syndromes in younger patients and hypoplastic myelodysplasia in older patients. These variations in clinical presentation and potential differences in pathophysiology between pediatric and adult patients with AA emphasize the importance of having data sets dedicated to pediatric cases on which to analysis patterns of presentation, diagnosis and treatment outcome and thereby recommend consensusdriven therapeutic algorithms, particularly in an environment in which there has been substantial historical variations in practice. ${ }^{2}$

The insights provided in the Rogers et al. analysis provides substantial clarity around several management issues in pediatric AA, but also pose several other ques-

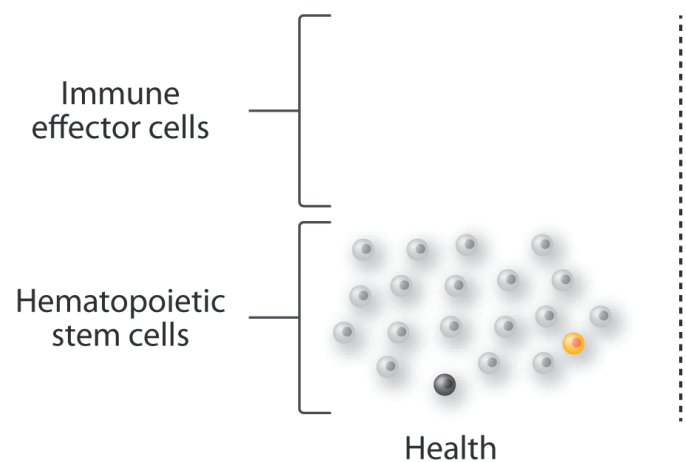

Treatment - IST or HCT

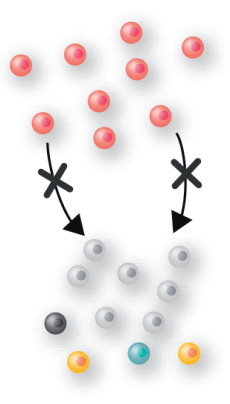

Aplastic anemia diagnosis

\section{Potential outcomes}

1) Complete response after IST

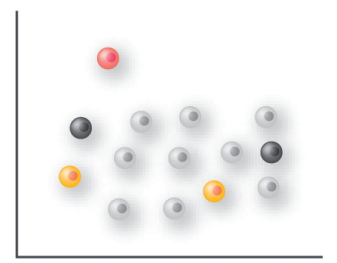

Slightly expanded clonal population within the HSC compartment

3 Clonal progression

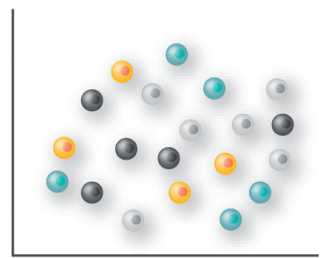

Expansion and emergence of new clonal populations may accompany progression to overt malignancy
2. Relapse after IST

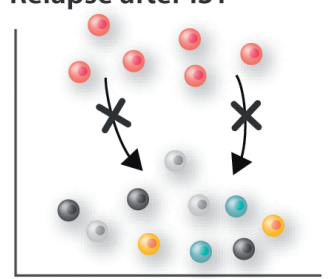

Expansion of clonal populations with survival advantage and resistance to immune attack

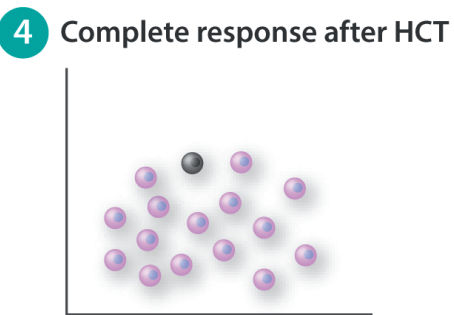

Engrafted donor HSC and quiescent abnormal HSC

Figure 1. Immune mediated attack on hematopoietic stem cells (HSC) and potential responses to therapy. IST: immunosuppressive therapy; HCT: hematopoietic stem cell transplant. 
tions either for ongoing analysis as their data set continues to expand and mature or as hypotheses to be tested in prospective studies.

A practical outcome of this consortium analysis is a real-world assessment of the applicability of the modified Camitta criteria for AA diagnosis first described in $1976 .^{3}$ These diagnostic criteria are still recommended by international guidelines for assessment of AA severity. ${ }^{4}$ In these criteria, in addition to the depth of marrow hypocellularity and peripheral blood cytopenias, a reticulocyte count is required both as a diagnostic criterion for $\mathrm{AA}$ and also to assist with severity classification. However, in a prior analysis by the NAPAAC, it was established that reticulocyte values substantially vary between institutions, making their inclusion in diagnostic criteria uncertain. ${ }^{2}$ To address this conundrum, Rogers et al. offer the interesting observation from their data set of a lack of correlation between hemoglobin and reticulocyte count in their pediatric cohort at the time of diagnosis, and suggest that hemoglobin may be a more accurate and clinically relevant parameter on which to base management decisions. Whilst the Camitta criteria have stood the test of time, and their use is a strong recommendation, some of their elements are based on relatively low quality C level source data. ${ }^{4}$ The findings outlined in the Rogers et al. paper re-iterate the importance of ongoing review and modification of diagnostic criteria as new data sets, such as that collated by the NAPAAC, come to hand.

Similarly, collation and description by co-operative groups of current patterns of clinical practice and the degree of its adherence to consensus guideline is an important element of continued improvement in practice, particularly for rare conditions where individual institutional experience may be limited. Currently, one of the most widely accepted management decisions in the treatment of young patients with AA is to offer HCT in patients aged under 40 years with an HLA matched sibling donor (MSD).$^{4-7}$ For those without a MSD, IST with anti-thymocyte globulin (ATG), most commonly horsederived, in combination with cyclosporine is used as initial therapy with HCT from unrelated donors (UD) reserved for those who do not respond or who relapse after IST. Of the cohort outlined in the Rogers et al. analysis, the majority of HCT undertaken as second-line therapy utilized UD, indicating the lack of a MSD for upfront use, and in those who eventually received second-line HSCT from a MSD, it is unclear why this donor was not used in the upfront setting. This particular question may be answerable in future analyses by the NAPAAC.

The Rogers et al. analysis demonstrated a striking difference in outcome following IST in pediatric patients compared to that in a historical cohort of adult patients. While complete response (CR) was only seen in $10 \%$ of adults treated with IST, ${ }^{8}$ the pediatric cohort showed CR rates of nearly $60 \%$. Despite this excellent response rate, a pattern of continual events, including death, relapse or transformation to hematologic malignancy following IST, resulted in a disappointing 5-year event-free survival (EFS) of $62 \%$, similar to the findings showing by Yoshida et $a l^{6}{ }^{6}$ The finding of a continued pattern of events even after apparent successful therapy with IST further rein- forces the view that normalization of peripheral blood parameters and marrow cellularity after immunosuppression does not imply normalization of hematopoietic clonality and/or immunological repertoire, and, as a consequence, the once-aplastic marrow remains at ongoing risk of recurrent aplasia, clonal evolution, and/or malignant transformation (Figure 1).

This description of the patterns of response and subsequent relapse (or other event) in pediatric AA raises at least three important questions. Firstly, what is the most appropriate salvage therapy for those patients relapsing after initial IST? Secondly, given the high rate of relapse/events, should HCT from any matched donor be considered as front-line therapy in children? Thirdly, are there better biomarkers under development that might provide greater guidance in the choice between these treatment options? With regards to the first question, the Rogers et al. paper provides clear guidance. Re-treatment was required in $35 \%$, and second-line therapy with an allogeneic HCT offered superior EFS to pursuing a second course of IST. These combined findings of unstable responses to IST, and the high rate of durable responses to HCT, contribute to the evolving debate as to whether HCT from any matched donor (related or unrelated) is preferable over IST as initial therapy for pediatric patients with proven AA. This question is clearly best answered in a randomized clinical trial, although such an undertaking would require a long-term commitment for feasible accrual and is only likely to succeed through consortia such as the NAPAAC. Lastly, biomarker development is critically required to more accurately determine the degree of clonal restriction (and therefore risk of clonal progression) and/or ongoing potential for immunological attack (and therefore post-IST relapse) to help determine whether IST or HCT should be offered as initial therapy. Clearly, in studies where restricted clonality is evident through genomic or cytogenetic analysis, poorer outcomes to IST are seen, indicating that with more sensitive techniques directed at assessment of the stem cell pool, more informed therapeutic decisions should follow. ${ }^{9}$ Again, it is likely that only through the co-ordinated efforts of consortia will sufficient biomarker samples be accumulated to begin to address this unmet need.

One clear determinant of clinical outcome from whatever therapy is chosen is the certainty with which the diagnosis is made. Increasingly, there is an appreciation that occult constitutional bone marrow failure syndromes may underlie what is thought to be a presentation of idiopathic AA, with significant implications for patient management. Through the increasingly readily available techniques for telomere length assessment ${ }^{10}$ and next generation sequencing for assessment of underlying germline lesions ${ }^{11}$ reclassification of many cases of $\mathrm{AA}$ is likely both during the prospective work up of new cases and retrospectively from archival diagnostic samples, which will further inform future treatment algorithms. As a greater clinical appreciation of the importance of diagnostic certainly has been met with greater diagnostic technical capacity, consensus recommendations increasingly incorporate evaluation of constitutional syndromes by chromosomal fragility testing in all AA patients presenting at younger than 50 years of age. Telomere length 
assessment is likely to be added to the routine work up panel in the near future. ${ }^{4}$ Reflecting these guidelines, Rogers et al. describe that while chromosomal fragility assessment was performed in most children, telomere length assessment was only performed at diagnosis in one-third of them.

Registries are crucial tools in efforts to improve outcomes for patients with rare diseases and their families. They serve as a means of pooling rare data in a standardized format in order to achieve meaningful sample sizes for subsequent analysis and allow comparison to historical or international cohorts, facilitate collaboration, generate hypotheses for future testing, and provide a framework for annotated sample collection and translational research. Further, participation in registry reporting contributes to achieving consistent and complete work up of new cases and provides a means of formulation and distribution of educational opportunities including multidisciplinary discussions which are so often needed in the management of rare conditions. Registries allow for the identification of patients, informing epidemiology assessments and areas of need, and may assist with allocation of scarce resources. Registries may facilitate feasibility assessments of and planning for clinical trials. The importance of registries focused on AA in particular is reflected in the increasing number of publications describing national outcome data in AA.,12-14 In this edition of Haematologica, Rogers et al. have made an important contribution to this data pool, informing optimal diagnostic and therapeutic approaches and, equally importantly, highlighting opportunities for further research and discussion in pediatric AA.

\section{Funding}

LF is supported by a Higher Degree Fellowship in Bone Marrow Failure from Maddie Riewoldt's Vision.

\section{References}

1. Rogers ZR, Nakano TA, Olson TS, et al. Immunosuppressive therapy for pediatric aplastic anemia: a North American Pediatric Aplastic Anemia Consortium study. Haematologica. 2019;104(10):1974-1983.

2. Williams DA, Bennett C, Bertuch A, et al. Diagnosis and treatment of pediatric acquired aplastic anemia (AAA): an initial survey of the North American Pediatric Aplastic Anemia Consortium (NAPAAC). Pediatr Blood Cancer. 2014;61(5):869-874.

3. Camitta BM, Thomas ED, Nathan DG, et al. Severe aplastic anemia: a prospective study of the effect of early marrow transplantation on acute mortality. Blood. 1976;48(1):63-70

4. Killick SB, Bown N, Cavenagh J, et al. Guidelines for the diagnosis and management of adult aplastic anaemia. Br J Haematol. 2016;172(2):187-207

5. Dufour C, Pillon M, Passweg J, et al. Outcome of aplastic anemia in adolescence: a survey of the Severe Aplastic Anemia Working Party of the European Group for Blood and Marrow Transplantation. Haematologica. 2014;99(10):1574-1581.

6. Yoshida N, Kobayashi R, Yabe H, et al. First-line treatment for severe aplastic anemia in children: bone marrow transplantation from a matched family donor versus immunosuppressive therapy. Haematologica. 2014;99(12):1784-1791.

7. Bacigalupo A. How I treat acquired aplastic anemia. Blood. 2017;129(11):1428-1436.

8. Townsley DM, Scheinberg P, Winkler T, et al. Eltrombopag Added to Standard Immunosuppression for Aplastic Anemia. N Engl J Med. 2017;376(16):1540-1550.

9. Yoshizato T, Dumitriu B, Hosokawa K, et al. Somatic Mutations and Clonal Hematopoiesis in Aplastic Anemia. $N$ Engl J Med. 2015;373(1):35-47.

10. Lai TP, Wright WE, Shay JW. Comparison of telomere length measurement methods. Philos Trans $\mathrm{R}$ Soc Lond B Biol Sci. 2018;373(1741).

11. Ghemlas I, Li H, Zlateska B, et al. Improving diagnostic precision, care and syndrome definitions using comprehensive next-generation sequencing for the inherited bone marrow failure syndromes. J Med Genet. 2015;52(9):575-584.

12. Contejean A, Resche-Rigon M, Tamburini J, et al. Aplastic anemia in the elderly: a nationwide survey on behalf of the French Reference Center for Aplastic Anemia. Haematologica. 2019;104(2):256-262.

13. Vaht K, Goransson M, Carlson K, et al. Incidence and outcome of acquired aplastic anemia: real-world data from patients diagnosed in Sweden from 2000-2011. Haematologica. 2017;102(10):1683-1690.

14. Zhu XF, He HL, Wang SQ, et al. Current Treatment Patterns of Aplastic Anemia in China: A Prospective Cohort Registry Study. Acta Haematol. 2019:1-9.

\title{
Mastering the multitude of monocytoses
}

\section{Ulrich Germing and Norbert Gattermann}

\author{
Department of Hematology, Oncology and Clinical Immunology, Heinrich Heine University Düsseldorf, Düsseldorf, Germany \\ E-mail: ULRICH GERMING - germing@med.uni-duesseldorf.de
}

doi:10.3324/haematol.2018.201053

I $\mathrm{n}$ this issue of the journal, Valent and coworkers report on diagnostic criteria for chronic myelomonocytic leukemia (CMML), CMML variants, and pre-CMML conditions. ${ }^{1}$ These CMML disorders have always been like orphans, trying to find their place in a suitable environment. They are rare entities but share chromosomal, molecular, morphological, hematologic, clinical, and prognostic features with other diseases in the large family of myeloid malignancies. The French-American-British group classified CMML as a myelodysplastic syndrome (MDS), based on its similarity to refractory anemia with excess blasts, although CMML "may have little in common with MDS showing trilineage dyspoiesis" ${ }^{2}$ and despite the fact that CMML features only minimal dysplasia in the erythroid lineage.
Pathologists and hematologists felt uncomfortable since there are more differences than similarities between CMML and MDS. World Health Organization classifications placed CMML in a "hermaphrodite" position between myeloproliferative neoplasms (MPN) and $\mathrm{MDS},{ }^{3-5}$ taking into consideration that there are proliferative characteristics as well as hematopoietic insufficiency associated with some degree of myelodysplasia. Despite all these efforts, CMML was once described as "lost in classification", as none of the classifications adequately reflects the marked heterogeneity of this group of myeloid neoplasms. ${ }^{6}$

A major problem with all myeloid neoplasias presenting without significant excess of blasts, in particular the cases presenting with monocytosis, is demarcation from reactive 